\title{
A Living Lab Approach for Enhancing Collaboration in Professional Communities
}

\author{
Hans Schaffers ${ }^{1}$, Steffen Budweg ${ }^{2}$, Kjetil Kristensen $^{1}$, Rudolf Ruland ${ }^{2}$ \\ ${ }^{1}$ ESoCE-NET, Via Cortina D'Ampezzo 164,00135 Rome, Italy, \{hschaffers, kristensen\}@esoce.net \\ ${ }^{2}$ Fraunhofer FIT, Schloss Birlinghoven, 53754 St. Augustin, Germany, \\ \{steffen.budweg,rudolf.ruland\}@fit.fraunhofer.de
}

\begin{abstract}
The ECOSPACE Integrated Project has developed an advanced collaborative workspace platform for eProfessionals. The technologies, services and collaboration tools have been developed, integrated and validated in various living labs addressing different contexts of eProfessional working. This paper reports about the Living Lab for Professional Communities of Innovation and covers three different user environments where innovative tools, services and practices have been experimented in different experimental settings. The first setting addresses the support of new work and collaboration practices in professional education. The second setting focuses on collaboration among business and research partners in promoting regional innovation. The third setting is about collaborative innovation in virtual communities. In the three settings, different user communities have adopted and experimented different sets of collaboration services. The paper presents the living lab methodology designed to launch, operate and monitor the different innovation and validation settings. Based on a cross-comparison of results and a longitudinal analysis, conclusions are drawn regarding the effectiveness of innovation services and collaborative platform, strategies to involve users and building user communities, and the effectiveness of the living labs methodology.
\end{abstract}

\section{Keywords}

Living labs, Collaboration, Innovation, Communities, Professionals, CSCW, User-centred design

\section{Introduction}

This paper evaluates the use of a living lab approach to the development of innovations in collaborative working environments for professional communities. It is based on selected results from the ECOSPACE Integrated Project (www.ip-ecospace.org), which aims to explore the vision of eProfessional collaborative working. This vision states that future knowledge workers will engage in seamless, dynamic and creative collaboration across teams, organizations and communities, through a personalised and interoperable collaborative work environment [Prinz et al 2006]. Professional Communities of Innovation are one of the three eProfessional domains targeted by ECOSPACE for exploration and validation of innovative collaboration services and tools; the other two domains are media creation and complex project management. For our chosen domain, the key objectives are to support community building and teamwork, to establish collaboration among professionals across boundaries of groups and organisations, and to improve the support for knowledge intensive collaboration tasks such as projects creation.

The general, well-known concept of a living lab involves the creation of a real-life, user-driven and open innovation environment. ECOSPACE implemented this approach by operationalising a living lab as a set of methodologies and tools to build the innovation environment and establish the living lab infrastructure, and to create and manage innovation projects in a specific context of user needs and organisations involved. This implies the situation-dependent use of a toolbox of mixed innovation and evaluation methods suited to the requirements and opportunities in particular circumstances. The key research question of this paper therefore is how the general living lab approach can be adapted to situational requirements and opportunities as given by the Professional Community of Innovation settings as described in this paper. 


\section{Related Work}

Building adequate technologies to support the collaboration of professionals both within and across organizational boundaries is a long-standing issue in multiple academic and professional domains. Different areas of research come together in living lab oriented work on collaborative work environments. Web 2.0 technologies such as shared workspaces, wikis, blogs and widgets are playing an increasingly important role in workplaces for professional communities resulting in new patterns of collaborative behaviour in networks and communities [Schaffers et al 2007]. User involvement throughout the design and development process in combination with organisational change approaches emerged from work on decision support systems [Keen and Scott Morton 1978], leading to evolutionary and participative design approaches [e.g. Ehn 1988] and has been taken up in action research [Baskerville 1999]. Cyclic and spiral approaches to software development have replaced the traditional waterfall methodologies. Approaches to usability engineering [e.g. Nielsen 1994] resulted in concrete methods for improved humancomputer interaction. Holistic and socio-technical systems oriented approaches towards work environments and information systems design have proposed to focus on the concept of work systems [Alter 2002] and socio-technical change [Lyytinen, Newman 2008]. Recent work on open innovation focused on the role of partnerships and open business models in the innovation process [Chesbrough 2006] and on radical ways of involving citizens [Von Hippel 2005].

The living lab concept embodies many of these elements but so far there have been no systematic attempts to integrate these concepts into a consistent set of living lab methodologies and expose such methodologies to empirical testing. This paper addresses this gap by providing case study experience, focusing on professional communities in education, research and innovation. Such professional communities are open networks, and this poses a number of challenges that should be explored and addressed to establish effective collaboration support in these complex and dynamic entities. Communication and collaboration patterns and platforms in these communities are often unstructured, heterogeneous and ill-defined. A useful categorisation of four basic collaboration modes was proposed by [Pisano and Verganti 2008]: 1) elite circle (closed network / hierarchical governance), 2) innovation mall (open network / hierarchical governance), 3) innovation community (open network / decentralized governance), and 4) consortium (closed network / decentralized governance). Innovation communities arguably constitute the most complex setting as neither participation nor governance is centrally coordinated. This has implications for how collaboration should be organized and supported, as the adoption of platforms are characterised more by brokering, trial and error and emerging practices than by managerial decisions.

In the following chapters we describe how the living lab approach has been used to establish an environment of user-driven innovation and to provide a project-based framework for initiating and developing innovation projects, resulting in enhanced collaboration tools and collaboration practices for professional communities of innovation.

\section{Methodology and Implementation}

\subsection{Overall approach}

As depicted in Fig. 1, the Living lab for Professional Communities of Innovation can be understood as a collection of value-adding activities resulting in innovations. Using the value chain concept [Porter 1985], operational and supporting activities are distinguished and presented as a living lab value chain. The living lab value system brings together innovation resources and organises innovation activities. Supporting activities include partnership development and maintenance, planning and project management, and maintaining the technical infrastructure. From this perspective emphasis is on creating, maintaining and enhancing the technical and organisational infrastructure for continuing innovation in the collaborative work 
environment for eProfessionals. This infrastructure includes the organisational, financial and collaborative arrangements among stakeholders ("business model"), the testbed infrastructure and access to user communities.

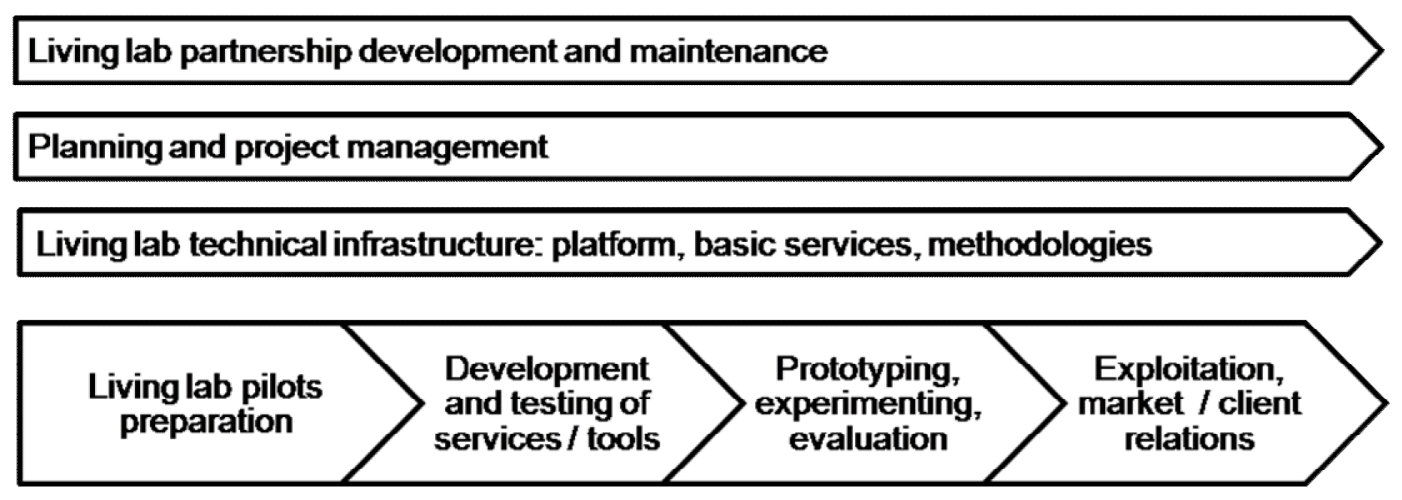

Fig. 1: Living lab as value system

Operational activities in the professional communities living lab focus on the specific innovation processes and outcomes. Such activities include the acquisition and organisation of the pilots, the preparation of pilots such as idea generation and selection of services and tools, the development and testing of services and tools, the user-driven prototyping and experimentation, and the exploitation of innovations into new business creation. During the operational activities, the cyclic and iterative process of innovation unfolds, supported by experimentation and observation methods and characterised by activities such as arranging user involvement for concrete experiments and validations, joint idea generation and service design, prototype development and testing, user training, small-scale user interaction and evaluations and wider scale deployment and use of the collaboration tools. Impacts and benefits are measured throughout the process.

\subsection{Living lab experimental settings}

The Professional Communities of Innovation living lab has launched and operated three experimental settings, reflecting different characteristics of such professional communities, to deploy and evaluate a series of specific innovations in the collaborative working environment of these communities. Besides, the ECOSPACE consortium has been acting as an "internal" experimental environment for tools in pre-mature stages of development. The three settings are:

- The AMI@Work community, hosted by the AMI portal and Fraunhofer FIT (www.amicommunities.eu), is a European-wide community of innovators, active in developing new research and innovation projects using a shared workspace. Thematically oriented subcommunities have evolved, including the CO-LLABS thematic network related to innovation support for SMEs and setting up new European-wide initiatives (www.amicommunities.eu/wiki/CO-LLABS). Within AMI and CO-LLABS we experimented a collaborative platform and various collaboration tools to support community building, communication and teamwork in the process of proposal development.

- The Frascati Living Lab community (bscw.frascatilivinglab.eu) has emerged within the Frascati region and is oriented towards business innovation and exploitation of technologies in areas such as space technologies and farming. It includes research institutes such as ESA-ESRIN, universities, small businesses, and innovation agencies such as BIC-Lazio, and it is also an active node of the Italian Network of Living Labs. Within the community we have introduced and experimented various collaboration tools focusing on portal services, shared workspaces, integrated upload and notify services, conferencing and community blogging.

- The 14Plus Living Lab was established to support the collaboration between the multiple stakeholders within the 14Plus project (www.projekt-14plus.de), promoting social and 
employment integration of young people above 14 years, and to cross-link schools, craft industries and local community partners. Different schools from several regions within the state of North Rhine-Westphalia in Germany are participating, as well as local and regional authorities and societal organisations. Within the 14Plus living lab setting, a series of collaboration services and tools have been deployed and tested, building on the web-based collaboration platform BSCW.

\subsection{Collaboration tools, scenarios and experimentation approach}

ECOSPACE has developed a collaborative working environment architecture and middleware service platform on the basis of which a large set of interoperable collaboration services and tools could be experimented and validated. After the services and tools pass the initial phase of technical testing, they enter the phase of validation, becoming candidates for deployment, use and adaptation in the living labs. The actual choice of services and tools is a result of matching the collaboration needs of users and the offerings of the tool developers. Table 1 presents the overview of collaboration tools experimented in the Professional Community Living Lab.

\begin{tabular}{|l|l|l|}
\hline Tool /System & Short description & Living lab Setting \\
\hline BSCW & Basic shared workspace system for collaborative working & 14Plus, Frascati, AMI \\
\hline Open ID & Single-Sign-On and Authentication Framework & 14Plus, Frascati, AMI \\
\hline Group Blog & Integration of blog software as own artefact into BSCW & 14Plus, Frascati, AMI \\
\hline Tagging & Tagging in relation to files and folders & 14Plus \\
\hline Portal modules & $\begin{array}{l}\text { Modular collaboration functionalities provided by UWA-compatible } \\
\text { (Netvibes, iGoogle) Widgets based on Flash/Flex and Javascript }\end{array}$ & 14Plus \\
\hline Wiki Portal & Integrated Wiki system with Single-Sign-On & 14Plus, AMI \\
\hline Presence & Workspace-integrated presence notification \& awareness support & 14Plus, Frascati \\
\hline Upload and notify & AJAX application to upload documents and notify user groups & Frascati, AMI \\
\hline Web conferencing & Web-based videoconferencing and shared applications (Marte) & Frascati, AMI \\
\hline Teambuilder & Creation of working groups based on competencies & Frascati, AMI \\
\hline $\begin{array}{l}\text { Workspace } \\
\text { synchronisation }\end{array}$ & Synchronisation of workspaces on different server via linking & Frascati, AMI \\
\hline Expert finder & Simple "expertise extractor" from documents and log files & Frascati \\
\hline Holmes & Extracting social networks using log files of shared workspaces & Frascati \\
\hline SIOC Xplore & Widget providing cross CWE-platform semantic querying & Frascati \\
\hline JammesWM & Mobile messaging and shared workspace management & Internal \\
\hline UncleShare & Annotation-based access control & Internal \\
\hline Smart Uploader & Intelligent document upload through distributed context information & Internal \\
\hline TM4Wiki & Topic map browser for ontology-aware repositories & Internal \\
\hline
\end{tabular}

Table 1: Overview on tools \& services within the Professional Communities Living Lab

The methodological framework used in carrying out these experimentation cycles is explained in [Löh et al 2009]. It combines two leading concepts. Experience Application Research [EC 2004] is promoting innovation projects based on user involvement in all stages of research, innovation and design. Action research [Baskerville 1999 and Schaffers et al 2008] is characterised by an interactive, cyclic and change oriented process approach to problem solving, covering phases of planning, actions, evaluation and learning, wherein all actors including users and researchers participate and learn. This combination fits very well the iterative nature of living labs experimentation, and with the characteristic of the living lab as a user-driven and participatory activity. Throughout the living lab experimentation, the development of scenarios has been important as an instrument to stimulate future thinking and challenge existing frames of mind regarding possible new community collaboration approaches, and to provide ideas to tool developers. We also used scenario development as a process of learning about bottlenecks and 
feasible ways of collaboration. The scenarios were not meant to be fully implemented in the living labs but rather as a communication instrument among actors involved: designers, researchers and users.

Within the framework of action research, living labs experimentation has partly followed a phased, cyclic and spiral development process that has been labelled Tool of the Month (ToM). A roadmap of tool introductions was elaborated and maintained, based on introducing, experimenting and evaluating innovative tools during a limited time period (mostly 2-4 months per tool). A snapshot of such ToM roadmap, used for Frascati and AMI settings, is depicted in Table 2. Activities carried out during one ToM cycle include: D: Define, prepare and plan experiments; P: Prepare technical availability of the tool; $\mathbf{C}$ : Communicate experiments to the target community; M: Develop Monitoring instruments; T: Provide Training to users; A:

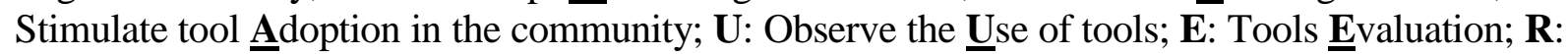

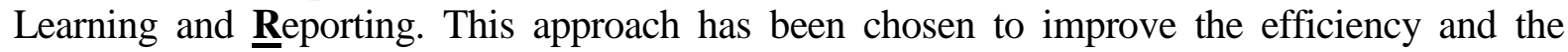
quality of the tool introduction process and create dedicated and situational living labs experiments.

\begin{tabular}{|l|l|l|l|l|l|l|l|l|l|l|l|l|l|l|l|l|l|}
\hline Tool & \multicolumn{3}{|l|}{ September 2008} & \multicolumn{4}{l|}{ October 2008 } & \multicolumn{4}{|l|}{ November 2008 } \\
\hline Week & 1 & 2 & 3 & 4 & 1 & 2 & 3 & 4 & 1 & 2 & 3 & 4 & 1 & 2 & 3 & 4 \\
\hline Group Blog & DT & P & C & AU & AU & AU & U & U & E & R & R & & & & & \\
\hline Upload/Notify & & & D & P & P & PC & AU & AU & U & U & U & R & R & & & \\
\hline Conferencing & & & & & D & P & P & P & PTU & PTU & PU & PU & CU & U & ER & \\
\hline Teambuilder & & & & & & & & & P & P & P & P & P & TA & U & U \\
\hline SIOC Xplore & & & & & & & & & & & & & D & P & P & P \\
\hline
\end{tabular}

Table 2: Tool experimentation roadmap in Frascati and AMI settings

\section{Living Lab Achievements}

One of the key achievements has been to set up the over-all joint living lab infrastructure, including the technical testbed, the launch and support of dedicated user communities, the process of generating and testing a continuous flow of service and tool concepts (Table 2), and the ability to initiate and operate experimentation and evaluation activities dedicated to the chosen settings. Training activities and surveys, polls and blogs have been conducted to support the living lab experiments and facilitate user-developer communication. For each experiment, events, processes and results were monitored. Overall, mixed results have been achieved and various lessons have been learned, which will be summarised in section 5. First, the next sections will provide a summary of achievements in the chosen settings.

\subsection{AMI@Work, CO-LLABS community}

The AMI@Work community has evolved during the last four years to more than 3000 members. During the first phase of ECOSPACE, the actual use of functionalities provided by the BSCW platform was monitored closely. In addition, user workshops were held, inviting community volunteers to experiment new functionalities such as presence, wiki and blogging, to discuss new ideas about collaboration within the community and providing tool experiments feedback to developers. Currently, a variety of new ECOSPACE-innovated BSCW services is in active use. In the later phase of ECOSPACE, a specific sub-community of AMI@Work was targeted, the CO-LLABS thematic network. In this setting, BSCW basic collaboration services and group blogging, the upload and notify service and web conferencing have been experimented. A core group of users has been using these functionalities actively, contributing valuable feedbacks to developers. Wider adoption within CO-LLABS has been proven to be more difficult. More comprehensive solutions to enable cross-workspace knowledge discovery and cross-community 
collaboration for new projects creation, based on workspace synchronisation and cross-platform semantic querying, are in the experimentation phase during Q2 2009.

\subsection{Frascati community}

A strong collaboration has emerged between ECOSPACE and the Frascati community, led by ESA-ESRIN. Living lab experimentation in the Frascati community concentrated on integrating the BSCW platform and the Frascati public living lab portal, and on specific tools such as group blogging, upload and notify, Teambuilder, and web-based video conferencing with application sharing. The tools were selected on the basis of maturity, usability and relevance for the community. At the level of platform and tools introduction and experimentation, results have been mixed. The BSCW platform has contributed considerably to establishing and strengthening the Frascati Community network. A core group of users has actively experimented the tools. However, it proved to be difficult adopt and experiment the newer tools in the wider community. Group blog has been integrated into the BSCW platform and can be considered as a "standard" functionality. It also has proven to be successful as a tool for user-developer communication. The upload and notify service has also been used in limited user settings and considerable feedback has been received by developers. Although promising and experimented successfully in limited user settings, technical work still has to be completed on Teambuilder and Web conferencing in order to deploy these tools successfully. However, these user experiments have resulted in many important feedbacks to ECOSPACE developers, technical as well as process related, and in more realistic ideas about user needs regarding collaborative support.

\subsection{Plus community}

The 14Plus Living Lab started in late 2006 and has been continued since. Collaboration tools and services have been developed targeting several challenges. These challenges included the provision of remote communication and collaboration among project partners; the need to address different working times and availabilities for meetings; the exchange, generalisation and re-use of work conducted in the partner schools; and the flexible integration of additional project partners from local communities and additional partner schools. An integrated collaborative work environment was developed based on the web-based groupware BSCW. Among the tools for living lab experimentation were the fully fledged document-exchange system, integrated Blogs and Wiki-systems, all accessible with a single sign-on. Additional services targeting awareness support for the presence of members, managing expectations on collaborative processes as well as sub-group awareness provided by activity folders were integrated within the platform, accompanied by tagging support, event notification and different collaboration widgets, allowing the integration of specific collaboration functionalities on external websites or portal pages (like iGoogle or NetVibes).

A continuous process of iterative feedback and development was initiated, supported by requirements collection through workshops, and by frequent demonstration and training activities. Adoption and adaptation of the tools in the work environment has been stimulated through the coaching activities of local champions within the 14Plus participating organisations. Based on our evaluations, it can be concluded that the 14Plus Living Lab has fulfilled the needs of the 14Plus community stakeholders to a considerable extent. For example, it has supported the distributed stakeholders with a CWE platform most intensively used for document management and efficient meeting preparation. Cooperation support was enhanced by introducing presence awareness functionalities, support for asynchronous communication and hand-overs. Additionally, the pilot schools were enabled to exchange the experiences and materials created or adopted. Indirect impacts evolved from making the work of the distributed communities and pilot schools visible and accountable, and by providing support for the monitoring, management and evaluation of the project. Finally, the 14Plus living lab reciprocally informed the iterative technology development and resulted in closer interaction and communication opportunities between technology developers and real-life users. 


\section{Discussion and Conclusions}

\subsection{The living lab concept}

The concept of living lab points to a collection of valuable concepts and processes, capable of attracting attention and mobilising discussions, but has not yet arrived in a phase of maturity and concreteness. There is not yet a systematic body of evidence on what works and what not, and in which context. There is still little experience on how living lab processes and methods can be successfully applied to the efficient and effective inception, development, adoption and experimentation of concrete innovations. In this context, the aim of our paper has been to contribute to living labs as concrete research and innovation method. Our living lab approach can be characterized as a multiple mixed-methods approach, taking into account the heterogeneous nature and complexity of the different communities, settings and stakeholders involved. Among the main characteristics of our approach are:

- A long-term approach to user involvement and experimentation, evaluating technologies throughout the life-cycle of real-life daily work as well as the project developments.

- A phased, cyclic approach to introduction, training, experimentation and evaluation of future collaboration technologies and services.

- A double-use technology strategy, applying new collaboration technologies for both the living lab communities themselves as well as for maintaining the overall living lab process, introduction, diffusion, training, evaluation and management to meet the challenge of distributed, asynchronous and virtual living lab settings [Budweg 2008].

\subsection{Some lessons learned}

Our CWE tools and technologies have been introduced and tested in contexts similar to real working situations. Training, demonstration and support activities have contributed to ECOSPACE living lab efforts to support end users offering tools that improve their condition, and soliciting user feedback that served to further improve these tools. As much as possible we have implemented training strategies as "learning by doing" and part of the appropriation and change process experienced by the users. Context-specific approaches are strongly preferable, together with local training support, preferably in the native tongue of the user community.

Disseminating training best practices between the various living lab settings has been a challenge due to inherent differences between the different living lab settings. One reflection is that training as a separate activity has been a moderately successful. Training is best performed by the tool owners together with scenario "owners", and in conjunction with broader initiatives.

The process of development, introduction, deployment and use has been monitored closely. An elaborated monitoring and evaluation approach has been set up and has been implemented partially. Large-scale living lab research projects require the involvement of social science experts as part of multi-disciplinary teams, balancing technical and social expertise.

Knowledge workers live busy lives. Engaging and invigorating external, dispersed user communities for volunteering to experiment and use our tools has been challenging, even in cases where the tools introduced had clear benefits compared to current ways of working. Overcoming initial change resistance towards "yet another tool" represents a challenge. Local community building represents a strong strategy for creating a user base, however even then a clear benefit needs to be presented to these users to convince them to participate in experiments.

The Tool of the Month approach requires a balance between offering new functionalities and managing the risk of saturation as new tools are added with similar (perceived or actual) benefits. In these situations, care should be taken to avoid cannibalisation effects.

The importance of usability cannot be overstated. In certain situations it has been difficult to sustain the use of tools beyond the initial testing stage. Equally, user engagement has been 
hindered because some of the tools were not sufficiently mature and needed additional technical testing. Thus, there is a clear limit to user-led co-creation.

\subsection{Workplace change}

ECOSPACE Living Labs provided us the environments to study changes in the eProfessional workplace (tools and work processes). In observing the living labs innovation process from the perspective of adoption and change, we aimed to study the success and failure factors and inhibitors and stimulators of workplace change.

It has been difficult to convince stakeholders to switch current tools to using the proposed BSCW-based working environment. Also, political sensitivities (roles and ambitions of important stakeholders in the regional innovation system) have played a role.

Again, establishing a collaborative culture at a practical level of joint development is a key bottleneck. Deployment of collaboration tools has focused on several levels: the support of a regional innovation breeding ground activity (pilots development), and supporting the lifecycle of project idea to realization.

\section{Acknowledgement}

This work has been partly funded by the European Commission through IST Project ECOSPACE (No. IST-2006-535208). The authors wish to acknowledge the Commission for their support and wish to acknowledge our gratitude and appreciation to ECOSPACE project partners contributing to developing ideas and concepts presented here.

\section{References}

Alter, S.: The work system method for understanding information systems and information systems research. Communications of the Association for Information Systems, Volume 9, pp 90-104, 2002.

Baskerville, R.: Investigating information systems with action research, Communications of the Association for Information Systems 2 (Article 19) (1999). Available at: http://cais.isworld.org.

Budweg, S. User Involvement and Co-Creation - Exploring Living Labs and Participatory Design in Distributed Contexts. Paper presented at the CHI 2008 WS Distributed Participatory Design, Florence, Italy, 2008.

Chesbrough, Henry: Open Business Models. Boston, MA: Harvard Business School Press, 2006.

Ehn, P.: Work-Oriented Design of Computer Artifacts. Hillsdale, NJ: Lawrence Erlbaum Associates, 1988.

European Communities (EC): ISTAG report on Experience and Application Research. Involving Users in the Development of Ambient Intelligence. Luxemburg, 2004.

Keen, P., Scott Morton, M.S.: Decision Support Systems. Addison Wesley, 1978.

Lyytinen, Kalle, Newman, Mike: Explaining information systems change: a punctuated socio-technical change model. European Journal of Information Systems 17, 589-613, 2008.

Löh, Hermann; Schaffers, Hans; Kristensen, Kjetil; Budweg, Steffen: From Theory to Practice. Making Product and Service Innovation Happen in CWE-related Living Labs. White paper, ECOSPACE, January 2009.

Nielsen, J.: Usability Engineering. Morgan Kaufmann, San Francisco, 1994.

Pisano, Gary; Verganti, Roberto: Which Kind of Collaboration is Right for You? Harvard Business Review. December, 2008.

Porter, Michael: Competitive Advantage. Free Press, 1985.

Prinz, W., Löh, H., Pallot, M., Schaffers, H., Skarmeta, A., Decker, S.: ECOSPACE - Towards an Integrated Collaboration Space for eProfessionals. CollaborateCom conference, Atlanta, November 2006.

Schaffers, H.; Slagter, R.; Kristensen, K.; Löh, H.: Web 2.0 Technologies and Workplace Paradigms to Enable eProfessional Workstyles. Proceedings of the 13th International Conference on Concurrent Enterprising, pp 155-162, 2007.

Schaffers, Hans; Garcia Guzman, Javier; Merz, Christian: An Action Research Approach to Rural Living Labs Innovation. In: P. Cunningham and M. Cunningham (Eds), Collaboration and the Knowledge Economy: Issues, Applications, Case Studies. IOS Press, pp 617-624, 2008.

Von Hippel, Eric: Democratizing Innovation. Cambridge, MIT Press, 2005. 